\title{
Outcomes, Measures and Recovery After Ambulatory Surgery and Anaesthesia: A review
}

\author{
Metha Brattwall • Margareta Warren-Stomberg • \\ Jan Jakobsson
}

Published online: 3 June 2014

(C) Springer Science + Business Media New York 2014

\begin{abstract}
Ambulatory surgery is becoming increasingly adopted and today more complex procedures and not only ASA 1-2 patients are scheduled to undergo surgery according to a fast track concept. Rapid recovery and resumption of capacity to stand, walk, void, eat and drink is essential for safe discharge. There is, however, an increasing need for better way to assess and measure recovery and outcome after surgery and anaesthesia. Morbidity and unplanned admission are no longer deemed adequate for assessing quality of performance or to use as tool to measure efforts to improve the medical care and or logistics. There are several aspects that should be considered when analysing the recovery process and measuring outcome after ambulatory surgery and anaesthesia. The Postoperative Quality of Recovery scale is a multidimensional tool that has been shown to be able to quantify and discriminate the recovery process. This review will provide an overview of recovery and outcome following ambulatory surgery/anaesthesia.
\end{abstract}

\section{Brattwall}

Department of Anaesthesiology \& Intensive Care, Institution for Clinical Sciences, Sahlgrenska Academy, 40530 Gothenburg, Sweden

\section{Warren-Stomberg}

Institute of Health and Care Sciences, The Sahlgrenska Academy, University of Gothenburg, 40530 Gothenburg, Sweden

\section{J. Jakobsson $(\bowtie)$}

Department of Anaesthesia \& Intensive Care, Institution for Clinical Science, Danderyds Hospital, Karolinska Institutet, 18288 Stockholm, Sweden

e-mail: jan.jakobsson@ki.se
Keywords Ambulatory surgery - Outcome - Recovery · Assessment

\section{Introduction}

Ambulatory surgery is increasing. There are several reasons for the increasing interest in and adoption of ambulatory surgery. The introduction of new less invasive surgical techniques, the increased understanding of the pathophysiology associated with the surgical trauma and thus general efforts to reduce the stress response associated with surgery are of importance. These factors have without a doubt had a major impact on the transition of in-hospital to ambulatory surgery for an increasing number of procedures. Minimal invasive surgery and tension free techniques have had an influence on the surgical stress as well as on postoperative pain. Modern anaesthesia/anaesthetic and postoperative pain management techniques have also contributed to a more rapid emergence from general anaesthesia. The multimodal or so called balanced analgesia regime has improved the recovery process and facilitates discharge with acceptable pain scores. Collaboration between surgeons, anaesthesiologists and nurses in the preoperative area as well as scrub nurses and postoperative unit personnel is also a factor of significant importance for efficient patient turnover. Fast tracking, enhanced recovery and early ambulation and discharge are nowadays concepts encountered in a majority of medical specialities. Enhanced recovery, early ambulation and discharge from hospital reduce the morbidity associated with bed rest such as thromboembolic and pulmonary complications. Reducing time in hospital may also reduce risk for hospital related infections and the risk in the elderly for cognitive impairment related to change in environment. 
The reduction in cost associated with reduced hospital stay is also of importance.

There are several aspects that need to be considered when recovery, follow-up and outcome associated with ambulatory surgery and anaesthesia are discussed. Major morbidity, mortality, unplanned hospital admission/prolonged hospital stay and readmission rate are traditional measures of outcome related to surgery in general but also to ambulatory anaesthesia/surgery.

There are also quality aspects that are commonly assessed. Pain and incidence and severity of postoperative nausea and vomiting (PONV) are patient factors of huge importance for quality of care.

Time events, emergence, time in recovery room and time to home-readiness and discharge are commonly used measures.

Assessment of quality of recovery after discharge; resumption of activities of daily living (ADL) is also increasingly used. The assessment of quality of recovery should also include assessment of emotional and cognitive performance.

American Association for Anaesthesiology has provided guidelines and a web-page covering papers around ambulatory anaesthesia http://www.asahq.org/coveo.aspx?q= Ambulatory\%20anaesthesia.

\section{Major Complications and Re-admission}

Major complications and morbidity are today rare events. There are several studies documenting most reassuring results. The classic study by Warner et al. [1] showed a most reassuring safety from the follow-up. A total of 38,598 patients aged 18 years and older undergoing 45,090 consecutive ambulatory procedures and anaesthetics were studied. Contact rates for $72 \mathrm{~h}$ and 30 days were 99.94 and $95.9 \%$, respectively. The complication rate was indeed low. Thirty-three patients either experienced major morbidity or died (1:1,366 [proportional risk]). Four patients died $(1: 11,273)$, two of myocardial infarction and two in automobile accidents. No patient died of a medical complication within 1 week of surgery. Of the 31 patients who developed a major morbidity $(1: 1,455), 14(45 \%)$ had myocardial infarction $(1: 3,220)$, seven $(23 \%)$ had a central nervous system deficit $(1: 6,441)$, five $(16 \%)$ had pulmonary embolism $(1: 9,018)$, and five $(16 \%)$ had respiratory failure $(1: 9,018)$. Four events $(13 \%)$ occurred within $8 \mathrm{~h}$ of surgery $(1: 11,273), 15(48 \%)$ in the next $40 \mathrm{~h}$ $(1: 3,006)$, and $12(39 \%)$ in the next 28 days $(1: 3,758)$.

Mezei and Chung [2] found similar outcome in a study from 1999. Preoperative, intraoperative and postoperative data were collected on 17,638 consecutive patients undergoing ambulatory surgery. One hundred ninety-three readmissions occurred within 30 days (readmission rate
$1.1 \%)$. Six patients returned to the emergency room, 178 patients were readmitted to the ambulatory surgical unit and 9 patients were readmitted as inpatients. Twenty-five readmissions were the result of surgical complications and one resulted from a pulmonary embolism. The complication-related readmission rate was $0.15 \%$ ( 1 in 678 procedures). No anaesthesia-related re-admissions or deaths were identified.

There are two more recent studies from Denmark assessing return to hospital following day surgery in the Copenhagen area.

Engbeak et al. [3] studied 16,048 patients who underwent 18,736 day surgery operations including 4,829 surgical abortions. Patients were retrospectively analysed for contacts to Danish hospitals within 60 post-operative days and the associated morbidity and mortality. Altogether 113 patients (not including the surgical abortions) were readmitted to hospitals with 117 complications definitely or likely related to day surgery. The most common complications were haematomas or haemorrhage $(0.40 \%)$ and infections $(0.29 \%)$. Morbidity after the two most common procedures, hernia repair and knee arthroscopy, was observed in 1:39 patients and 1:220 patients, respectively. More serious complications included four patients with septic arthritis of the knee and six patients with venous thromboembolism. After surgical abortion, pelvic inflammation and bleeding were observed in 3.1 and $2.2 \%$, respectively, with centre differences. Altogether no myocardial infarctions, central nervous system deficits, pneumonias or deaths were recorded that could definitely or likely be related to day surgery. Majholm et al. [4••] prospectively recorded data from 57,709 day surgical procedures performed in eight day surgery centres over a 3-year period. The overall rate of return to hospital visits was $1.21 \%$ [95 \% confidence interval (CI) $1.12-1.30 \%$ ] caused by a wide range of diagnoses. No deaths were definitely related to the surgical procedure. The most common return to hospital visits were due to haemorrhage/ haematoma $0.50 \%$ (95\% CI $0.44-0.56 \%)$, infection $0.44 \%(95 \%$ CI $0.38-0.49 \%)$ and thromboembolic events $0.03 \%$. Major morbidity was rare. Procedures with the highest rate of complication were tonsillectomies $11.4 \%$, surgically induced abortions $3.13 \%$ and inguinal hernia repairs $1.23 \%$.

There is an increase in obese patients and Joshi et al. [5] published in November 2013 a systematic review of studies published between 1948 and May 2012, assessing perioperative outcome in adult obese patients undergoing ambulatory surgery. A total of 106,119 patients were included in the analysis with 62,476 patients included in the prospective trials and 43,643 patients included in the retrospective trials. Of these, 39,548 patients underwent bariatric surgery. The super obese (body mass index $[\mathrm{BMI}]>50 \mathrm{~kg} / \mathrm{m}$ ) 
appeared to be at higher risk of complications. Still they did not find overall any major increased risk for unplanned admission. The lack of increase in unanticipated admission rate in this patient population may of course be related to thorough preoperative assessment and avoidance of patients with co-morbid conditions.

Mathis et al. [6•] conducted a multi centre study looking at morbidity or mortality within $72 \mathrm{~h}$. Intraoperative complications included adverse cardiovascular events; postoperative complications included surgical, anaesthetic, and medical adverse events. They found a reassuringly low complication rate. Of 244,397 surgeries studied, $232(0.1 \%)$ experienced early perioperative morbidity or mortality. They found overweight being one out of increased risk factors along with chronic obstructive pulmonary disease, history of transient ischaemic attack/stroke, hypertension, previous cardiac surgical intervention, and prolonged operative time.

The data around morbidity and need for return to hospital following ambulatory surgery should be put into the perspective of recent studies on re-admission following inhospital major surgery. There are 2 recent papers on inhospital surgery looking at readmission with a time window analysed readmission within 30-days. Kohlnhofer et al. [7] collected data on patients undergoing inpatient general surgery procedures, institutional National Surgical Quality Improvement Project database from 2006 to 2011. They found $9 \%$ of patients readmitted within 30 days after discharge. Multivariable analysis demonstrated age, dyspnoea, and American Society of Anesthesiologists class to be independent risk factors for readmission. In addition, patients who suffered from multiple complications had a decreased risk for readmission as length of stay increased. Patients with $<2$ postoperative complications had an increased risk for readmission as length of stay increased.

Kassin et al. [8] collected data at a single academic centre between 2009 and 2011. They found that the common reasons for readmission were gastrointestinal problem/complication (27.6\%), surgical infection (22.1\%) and failure to thrive/malnutrition (10.4\%). Co-morbidities associated with risk of readmission included disseminated cancer, dyspnoea and preoperative open wound $(P<0.05$ for all variables). Surgical procedures associated with higher rates of readmission included pancreatectomy, colectomy and liver resection. Postoperative occurrences leading to increased risk of readmission were blood transfusion, postoperative pulmonary complication, wound complication, sepsis/shock, urinary tract infection and vascular complications.

\section{Outcome, the Recovery Process}

There is no unequivocal definition of postoperative recovery. Outcome must be assessed not only by major morbidity, admission and readmission but by analysis of recovery, resumption of capacities following surgery and anaesthesia. The recovery process is complex and involves not only the elimination of anaesthetics and associated administered drug effects but also a variety of aspects triggered by surgery, the surgical trauma and potential secondary effects caused by the anaesthetic drugs administered. Pain, PONV, recovery of ADL, becoming street fit, full restitution of physical and cognitive capacity are all factors that should somehow be assessed after surgery and anaesthesia. Surgical impacts on health-related Quality of Life (HRQoL) and patients' satisfactions are also variables that should be taken into account. Recovery is complex, and there are still limited studies as well as well-defined tools for their assessment.

\section{Impact of Anaesthetic Technique}

There are several anaesthetic techniques used for ambulatory surgery. All are seemingly safe and efficacious, and there are limited data showing that there are any significant differences by one or the other [9]. Indeed, the risk for major morbidity and mortality is low in conjunction to ambulatory anaesthesia and the most recent meta-analysis by Guay et al. [10••] published in 2014 could not find hard evidence for major protecting properties of one anaesthetic technique over the other in general. The authors merely concluded that compared with general anaesthesia, a central neuraxial block may reduce the 0-30-day mortality for patients undergoing surgery with intermediate to high cardiac risk (level of evidence, moderate). Gupta et al. [11] conducted a systematic review published in 2004. They found small differences and concluded that the choice of the main anaesthetic should be guided by the experiences of the individual physician. The routines and equipment at the department are also of importance. The specific anaesthetic had only minor role in outcome after ambulatory surgery. There is a recent meta-analysis done by Vaughan et al. [12•] around anaesthetic technique for elective laparoscopic cholecystectomy and outcome, showing a similar result. They found no clear evidence of a difference in the proportion of patients who were discharged on the same day between any of the comparisons. Overall, 472/554 patients $(85 \%)$ included in the review were discharged on the day of surgery. Return to activity and return to work or any quality of life measure were not reported in any of the trials.

Emergence-Awakening from Anaesthesia

There are studies showing differences in emergence of desflurane-based anaesthesia providing the most rapid and predictable emergence [13, 14, 15]. Likewise Liu [16] 
conducted a meta-analysis showing beneficial effects from the drug titration by BIS-monitoring. He found that the use of BIS monitoring reduced anaesthetic consumption, risk of nausea and vomiting and recovery room time; however, these benefits did not reduce time spent in the ambulatory surgery unit. The early recovery may also be assessed by the classical Aldrete [17] score as well as the possibility to by-pass the recovery room altogether and transporting the patient directly to a low dependency facility. Both White et al. [18] and Fanelli et al. [19] found desflurane and sevoflurane based ambulatory anaesthesia to provide an equally high number of patients eligible for fast-tracking through by-passing the recovery room.

\section{Impact of Pain Management}

The positive effects of multi-modal or balanced postoperative analgesia were shown decades ago and have become standard of care for ambulatory surgery [20, 21]. The combination of local anaesthesia, acetaminophen, NSAIDs and the lowest effective dose of opioid have reduced the well-known side-effects associated with opioid analgesics and is today a well-established practice [22]. White et al. [23] compared celecoxib and ibuprofen in a placebo-controlled study and showed both substances to have a positive effect on recovery. Both ibuprofen $(1,200 \mathrm{mg} /$ day $)$ and celecoxib (400 mg/day) significantly decreased the need for rescue analgesic medication in the early post-discharge period, leading to an improvement in the quality of recovery and patient satisfaction with their pain management after outpatient surgery. The Oxford league table of analgesics in acute pain provides a comprehensive overview of most common oral analgesics. ${ }^{1}$

\section{Impact of PONV}

PONV, the little big problem has major impact on quality of care and time to discharge [24]. Risk scoring and risk score-based multi-modal PONV prophylaxis is today commonly adopted [25]. The adoption of risk scoring and subsequent multi-modal PONV prophylaxis is not a guarantee for the avoidance of emetic symptoms [26]. Efforts are warranted in order to improve the prevention and treatment of PONV reducing the early as well as late risks for emetic sequelae? [27]. There are new consensus guidelines providing update around risk assessment and strategies for prevention and treatment of both early and delayed PONV $[28 \bullet \bullet$.

\footnotetext{
${ }^{1}$ http://www.medicine.ox.ac.uk/bandolier/booth/painpag/Acutrev/ Analgesics/Leagtab.html
}

Resumption of ADL

Wong et al. [29] described in 2009 the Functional Recovery Index, a simple and easy to use tool for assessment of functional recovery. The 14 simple and straightforward questions around every day living activities with a response scale ranging from; Not difficulty at all toExtremely difficult was shown to have excellent reliability, good validity, responsiveness and acceptability. The authors suggest this test to be a good instrument for assessing functional recovery of ambulatory surgical patients. Studies about the subjective experience are sparse. Berg et al. [30] asked patients about their perception of the recovery following surgery/anaesthesia they found that patients commonly experiences a comprising of mixed internal and external prerequisites; changes in ordinary life and an extensive shift about responsibility at home. Pakzad et al. [31] suggested that more extensive information and 'education' could be of value.

\section{The Postoperative Recovery Scales}

There are several aspects that need to be assessed in order to describe the recovery process after surgery and anaesthesia. During the last decades a number of recovery assessment tools have been introduced. Herra et al. [32] conducted a systematic review of postoperative recovery outcome measurements after ambulatory surgery in 2007 . They assessed available tool from eight dimensions and concluded that only one instrument, the 40-item Quality of recovery score, fulfilled all the eight criteria. It was, however, commented that this instrument was not specifically designed for ambulatory surgery and anaesthesia. Stark et al. [33] showed that a condensed version of the QoR 40 score, the Quality of Recovery-15 provides a valid, extensive, and yet efficient evaluation of postoperative recovery. The recovery scores are constructed on repeated measures of various domains, with base-line preoperative performance as the level of performance that should be regained in order to fulfil the definition of "recovered". There are of course several pitfalls with repeated tests, possibility of learning effect, low level bias because of bad initial performance and ceiling bias because the test does not provide sufficient strength to document postoperative changes. Bost et al. compared the numeric rating scale (NRS) pain score, the 8-item Short-Form Health Survey (SF-8), and the 40-item Quality of Recovery from Anaesthesia (QoR) during the first 4 days after ambulatory knee surgery with regional anaesthesia. They found weaknesses with all the scales and suggested that a composite was needed to gain a full recovery profile. DeVine et al. conducted a meta-analysis aiming at evaluating the correlation and responsiveness of patient-reported pain with function 
and quality-of-life outcomes after spine surgery. They observed little correlation between the change in pain and the change in HRQoL outcome measures. They suggest that when evaluating the surgical outcomes for CLBP in the clinical-research setting, a shorter version for measuring general HRQoL (e.g. SF-12, EQ-5D) to minimize clinician and patient burden should be used. The Postoperative Quality of Recovery scale PQRS was introduced and validated in 2010 [34••]. It is a 6-dimensional repeated performance recovery assessment tool. It assess six domains of recovery; physiologic, nociceptive, emotive, ADL, cognitive, and overall patient perspective. Baseline testing in all domains is performed between 1 and 14 days preoperatively. Early measurement is performed at $40 \mathrm{~min}\left(T_{40}\right)$ after cessation of anaesthesia and is principally designed to assess recovery at the point of discharge from the postoperative anaesthesia care unit. Late recovery refers to the measurements performed in the first week after surgery at 1 and 3 days postoperatively $\left(D_{1}\right.$ and $\left.D_{3}\right)$. Longterm recovery may be assessed at 3 months postoperatively $\left(M_{3}\right)$. In late and long-term measurements, the focus changes from basic physiologic and home-readiness recovery to cognitive recovery and return to previous or expected level of functioning at home or workplace. The PQRS has been shown to be easy to use clinically and a sensitive measure of the recovery process being able to differentiate between common procedures such as tonsillectomy and nasal surgery [35].

\section{Persistent Postoperative Pain}

Long-lasting pain following surgery is also one outcome variable that should be taken into account following ambulatory surgery. Long-lasting, persistent postsurgical pain (PPP) is a major concern [36] and has been reported following breast cancer surgery (BCS), groin hernia repair (GHR), and lung cancer surgery (LCS) in 13, 2, and 4-12\%, respectively [37]. Attempts have been made in order to identify risk factors and subsequent interventions to minimise the risk for long-lasting pain. Aasvang et al. [38] found in a recent study that $12.4 \%$ had "moderate/ severe" PPP at 6 months among patients that had undergone open or laparoscopic transabdominal preperitoneal elective GHR. Logistic regression analysis identified four risk factors for PPP: high preoperative Activity Assessment Scale (AAS) score, preoperative pain to tonic heat stimulation, 30-day postoperative pain intensity and sensory dysfunction in the groin at 6 months (nerve damage) (all $P<0.03$ ). A risk prediction model of only preoperative factors and choice of surgical technique revealed increased preoperative AAS score, increased preoperative pain to heat stimulation, and open surgery to increase the risk for PPP (all $P<0.02$ ). There is a need for standardisation, structured data collection and potential for a common data base in order to better understand the core risk associated to the development of long-lasting pain after surgery [39]. This is in line with the Cochrane review by Caparro et al. [40] who reviewed 40 randomized controlled trials and warranted additional evidence from more well-designed, large-scale trials in order to more rigorously evaluate pharmacological interventions for the prevention of chronic pain after surgery.

\section{Neurocognitive Effects of Surgery and Anaesthesia}

Surgery triggers a neuro-inflammatory response that may cause prolonged effects on cognitive functions. Postoperative cognitive dysfunction is not uncommonly seen in the elderly following major surgical trauma. The neuro-inflammatory response has been suggested to be one of major factors influencing the occurrence of POCD. Experimental data suggest that the neuro-inflammatory response to surgery triggers also changes in the younger brain [41]. The potential benefit from EEG based depth-of-anaesthesia monitoring in reducing the risk early as well as protracted cognitive dysfunction has been suggested. Jildenstål et al. [42] found AAItitrated (the use of a depth of anaesthesia monitor based on processed auditory evoked potential technique) desflurane anaesthesia to reduce the incidence of low cognitive test performance at 24-h after ophthalmologic day surgery. Chan et al. [43•] have shown similar beneficial effects from BIStitrated anaesthesia in elderly undergoing major surgery, a reduced risk for postoperative delirium and cognitive decline. Their study results would translate into that for every 1,000 elderly patients undergoing major surgery, anaesthetic delivery titrated to a range of BIS between 40 and 60 would prevent 23 patients from POCD and 83 patients from delirium. Whether these findings are applicable also to elderly undergoing ambulatory procedures warrants further studies.

\section{Ultimate Health-Related Quality of Life Measures}

Broad general tools for the assessment of HRQoL have been used and should be considered alternatives for the assessment of outcome. The SF-36 is a multi-purpose, short-form health survey with only 36 questions. ${ }^{2}$ It yields an 8-scale profile of functional health and well-being scores as well as psychometrically based physical and mental health summary measures and a preference-based health utility index. It is a generic measure, as opposed to one that targets a specific age, disease or treatment group. Accordingly, the SF-36 has proven useful in surveys of general and specific populations, comparing the relative burden of diseases, and in differentiating the health benefits

\footnotetext{
${ }^{2}$ http://www.sf-36.org/tools/sf36.shtml
} 
produced by a wide range of different treatments. The EQ$5 \mathrm{D}^{3}$ is another well-validated general health assessment tool. The EQ-5D-3L essentially consists of 2 pages-the EQ-5D descriptive system (page 2) and the EQ visual analogue scale (EQ VAS) (page 3). The EQ-5D-3L descriptive system comprises the following 5 dimensions: mobility, self-care, usual activities, pain/discomfort and anxiety/depression. Each dimension has 3 levels: no problems, some problems, extreme problems. The Euro Quality 5-dimension score EQ-5D has been used by Brattwall et al. [44]. They found procedure-specific changes in the postoperative health profile after day surgery. Specifically arthroscopy patients recovered more slowly compared with inguinal hernia repair and cosmetic breast surgery patients. It was concluded that the time for final evaluation differs significantly between procedures. Velanovich [45] used the SF-36 when assessing the health impact difference between open and laparoscopic surgery. $\mathrm{He}$ found that the laparoscopic technique was associated to better quality-of-life outcomes than open surgery for cholecystectomy, splenectomy and oesophageal surgery. However, open hernioplasty has at least as good, if not better, health status outcomes than laparoscopic repair. Dhankhar et al. [46] used SF-36 looking for differences between two hernia repair techniques made under local versus general anaesthesia. They showed that Lichtenstein repair under local anaesthesia is as good as totally extraperitoneal repair under general anaesthesia.

\section{Summary and Conclusion}

Outcome of anaesthesia is not well defined. Basic requirements are adequate intraoperative anaesthesia and safety. Awareness with recall is the most rare but catastrophic event [47]. Major morbidity and mortality associated to ambulatory surgery and anaesthesia is rare. It is hard to predict whether we will see an increase in morbidity and re-admission to hospital as ambulatory surgery is increasingly adopted also for the elderly and more fragile patients as well as being utilized with more extensive surgical procedures.

There is a growing interest in whether there are differences between anaesthetics with regards to their potential risk for neurotoxicity in the developing brain, when used in neonates and especially in pre-term neonates. There are an increasing number of studies evaluating the development of the subtle but annoying experience of Postoperative Cognitive Dysfunction [48]. POCD is most commonly seen in the elderly and follows major surgery and it has been suggested that ambulatory surgery and enhanced recovery pathway may be associated with less cognitive impairment

\footnotetext{
${ }^{3}$ http://www.euroqol.org/about-eq-5d/how-to-use-eq-5d.html
}

as compared to traditional in-hospital care $[49,50]$. There is likewise an interest in if and how anaesthetics and POCD can eventually impact the processes associated with dementia [51].

Recent interest in whether anaesthetic technique and influence in immune response could have an impact on the risk for cancer recurrence has emerged. It seems reasonable to conclude that there is still lack of robust Clinical data to assess and change practice [52,53]. There is also a debate around whether anaesthetics e.g. halogenated inhaled anaesthetics could have protecting effects, ameliorating the ischaemia reperfusion injury [54].

Outcome and recovery associated to ambulatory anaesthesia is still much based on time events and logistics and subsequent economical and health care resource utilisation; emergence/awakening characteristics having an impact on theatre turnover. The benefits of rapid awakening and resumption of protecting reflexes should, however, not be neglected from a safety perspective. Fast-tracking meaning the possibility to by-pass the recovery room high dependency area is also of interest. Time to discharge and unplanned hospital admissions are important factors relating both to obvious factors such as presence of pain and PONV but also to other practicalities such as timing for the escort.

Recovery in a more protracted perspective how patients resume ADL, is of increasing interest and tools like the PQRS provide a simple and validated option. HRQoL measures such as the EQ-5D and the SF-36 questionnaires are tools to be used in order to evaluate new surgery and anaesthetic techniques and major changes in practice.

\section{Compliance with Ethics Guidelines}

Conflict of Interest Jan Jakobsson has received financial support through grants from Baxter, Abbott Laboratories, Pfizer, Mundipharma, MSD, and Linde, and has also received compensation for service as a consultant from Pfizer, MSD, and Linde. Metha Brattwall and Margareta Warren-Stomberg declare that they have no conflict of interest.

Human and Animal Rights and Informed Consent This article does not contain any studies with human or animal subjects performed by any of the authors.

\section{References}

Papers of particular interest, published recently, have been highlighted as:

- Of importance

•• Of major importance

1. Warner MA, Shields SE, Chute CG. Major morbidity and mortality within 1 month of ambulatory surgery and anesthesia. JAMA. 1993;270:1437-41. 
2. Mezei G, Chung F. Return hospital visits and hospital readmissions after ambulatory surgery. Ann Surg. 1999;230:721-7.

3. Engbaek J, Bartholdy J, Hjorts $\varnothing$ NC. Return hospital visits and morbidity within 60 days after day surgery: a retrospective study of 18,736 day surgical procedures. Acta Anaesthesiol Scand. 2006;50:911-9.

4. • Majholm B, Engbæk J, Bartholdy J, Oerding H, Ahlburg P, Ulrik AM, Bill L, Langfrits CS, Møller AM. Is day surgery safe? A Danish multicentre study of morbidity after 57,709 day surgery procedures. Acta Anaesthesiol Scand. 2012;56:323-31. A paper describing return to hospital after day surgery in Denmark, almost 58000 procedures were evaluated.

5. Joshi GP, Ahmad S, Riad W, Eckert S, Chung F. Selection of obese patients undergoing ambulatory surgery: a systematic review of the literature. Anesth Analg. 2013;117:1082-91.

6. - Mathis MR, Naughton NN, Shanks AM, Freundlich RE, Pannucci CJ, Chu Y, Haus J, Morris M, Kheterpal S. Patient selection for day case-eligible surgery: identifying those at high risk for major complications. Anesthesiology. 2013;119:1310-21. A huge multi-center study looking at risks factors associated to early complications following day surgery.

7. Kohlnhofer BM, Tevis SE, Weber SM, Kennedy GD. Multiple complications and short length of stay are associated with postoperative readmissions. Am J Surg. 2014;207(4):449-56. doi:10. 1016/j.amjsurg.2013.10.022.

8. Kassin MT, Owen RM, Perez SD, Leeds I, Cox JC, Schnier K, Sadiraj V, Sweeney JF. Risk factors for 30-day hospital readmission among general surgery patients. J Am Coll Surg. 2012;215:322-30.

9. Pollard BJ, Elliott RA, Moore EW. Anaesthetic agents in adult day case surgery. Eur J Anaesthesiol. 2003;20:1-9.

10. •• Guay J, Choi P, Suresh S, Albert N, Kopp S, Pace NL. Neuraxial blockade for the prevention of postoperative mortality and major morbidity: an overview of Cochrane systematic reviews. Cochrane Database Syst Rev. 2014;1:CD010108. doi: 10.1002/14651858.CD010108.pub2. A systematic review around the effects of regional anaesthesia on mortality and major morbity.

11. Gupta A, Stierer T, Zuckerman R, Sakima N, Parker SD, Fleisher LA. Comparison of recovery profile after ambulatory anesthesia with propofol, isoflurane, sevoflurane and desflurane: a systematic review. Anesth Analg. 2004;98:632-41.

12. • Vaughan J, Nagendran M, Cooper J, Davidson BR, Gurusamy KS. Anaesthetic regimens for day-procedure laparoscopic cholecystectomy. Cochrane Database Syst Rev. 2014;1:CD009784. doi:10.1002/14651858.CD009784.pub2. A review around the impact of anaesthtic regime on outcome following day-case laparoscopic choloecystectomy.

13. Wachtel RE, Dexter F, Epstein RH, Ledolter J. Meta-analysis of desflurane and propofol average times and variability in times to extubation and following commands. Can $\mathrm{J}$ Anaesth. 2011;58:714-24.

14. Dexter F, Bayman EO, Epstein RH. Statistical modeling of average and variability of time to extubation for meta-analysis comparing desflurane to sevoflurane. Anesth Analg. 2010;110:570-80.

15. Jakobsson J. Desflurane: a clinical update of a third-generation inhaled anaesthetic. Acta Anaesthesiol Scand. 2012;56:420-32.

16. Liu SS. Effects of Bispectral Index monitoring on ambulatory anesthesia: a meta-analysis of randomized controlled trials and a cost analysis. Anesthesiology. 2004;101:311-5.

17. Kaur A, Jain AK, Sehgal R, Sood J. Hemodynamics and early recovery characteristics of desflurane versus sevoflurane in bariatric surgery. J Anaesthesiol Clin Pharmacol. 2013;29:36-40.

18. White PF, Tang J, Wender RH, Yumul R, Stokes OJ, Sloninsky A, Naruse R, Kariger R, Norel E, Mandel S, Webb T, Zaentz A.
Desflurane versus sevoflurane for maintenance of outpatient anesthesia: the effect on early versus late recovery and perioperative coughing. Anesth Analg. 2009;109:387-93.

19. Fanelli G, Berti M, Casati A. Fast-track anaesthesia for laparoscopic cholecystectomy: a prospective, randomized, multicentre, blind comparison of desflurane-remifentanil or sevoflurane-remifentanil. Eur J Anaesthesiol. 2006;23:861-8.

20. Eriksson H, Tenhunen A, Korttila K. Balanced analgesia improves recovery and outcome after outpatient tubal ligation. Acta Anaesthesiol Scand. 1996;40(2):151-5.

21. Michaloliakou C, Chung F, Sharma S. Preoperative multimodal analgesia facilitates recovery after ambulatory laparoscopic cholecystectomy. Anesth Analg. 1996;82(1):44-51.

22. Wickerts L, WarrénStomberg M, Brattwall M, Jakobsson J. Coxibs: is there a benefit when compared to traditional nonselective NSAIDs in postoperative pain management? Minerva Anestesiol. 2011;77(11):1084-98.

23. White PF, Tang J, Wender RH, Zhao M, Time M, Zaentz A, Yumul R, Sloninsky A, Naruse R, Kariger R, Webb T, Fermelia $\mathrm{DE}$, Tsushima GK. The effects of oral ibuprofen and celecoxib in preventing pain, improving recovery outcomes and patient satisfaction after ambulatory surgery. Anesth Analg. 2011;112(2):323-9.

24. Myklejord DJ, Yao L, Liang H, Glurich I. Consensus guideline adoption for managing postoperative nausea and vomiting. WMJ. 2012;111(5):207-13.

25. Fero KE, Jalota L, Hornuss C, Apfel CC. Pharmacologic management of postoperative nausea and vomiting. Expert Opin Pharmacother. 2011;12(15):2283-96.

26. White PF, O'Hara JF, Roberson CR, Wender RH, Candiotti KA, POST-OP Study Group. The impact of current antiemetic practices on patient outcomes: a prospective study on high-risk patients. Anesth Analg. 2008;107(2):452-8.

27. Hache JJ, Vallejo MC, Waters JH, Williams BA. Aprepitant in a multimodal approach for prevention of postoperative nausea and vomiting in high-risk patients: is there such a thing as "too many modalities"? ScientificWorldJournal. 2009;28(9):291-9.

28. • Gan TJ, Diemunsch P, Habib AS, Kovac A, Kranke P, Meyer TA, Watcha M, Chung F, Angus S, Apfel CC, Bergese SD, Candiotti KA, Chan MT, Davis PJ, Hooper VD, Lagoo-Deenadayalan S, Myles P, Nezat G, Philip BK, Tramèr MR; Society for Ambulatory Anesthesia. Consensus guidelines for the management of postoperative nausea and vomiting. Anesth Analg. 2014;118(1):85-113. A Consensus paper around the management of PONV.

29. Wong J, Tong D, De Silva Y, Abrishami A, Chung F. Development of the functional recovery index for ambulatory surgery and anesthesia. Anesthesiology. 2009;110(3):596-602.

30. Berg K, Årestedt K, Kjellgren K. Postoperative recovery from the perspective of day surgery patients: a phenomenographic study. Int J Nurs Stud. 2013;50(12):1630-8. doi:10.1016/j.ijnurstu.2013. 05.002 .

31. Pakzad H, Thevendran G, Penner MJ, Qian H, Younger A. Factors associated with longer length of hospital stay after primary elective ankle surgery for end-stage ankle arthritis. J Bone Joint Surg Am. 2014;96(1):32-9. doi:10.2106/JBJS.K.00834.

32. Herrera FJ, Wong J, Chung F. A systematic review of postoperative recovery outcomes measurements after ambulatory surgery. Anesth Analg. 2007;105(1):63-9.

33. Stark PA, Myles PS, Burke JA. Development and psychometric evaluation of a postoperative quality of recovery score: the QoR15. Anesthesiology. 2013;118(6):1332-40.

34. $\bullet$ Royse CF, Newman S, Chung F, Stygall J, McKay RE, Boldt J, Servin FS, Hurtado I, Hannallah R, Yu B, Wilkinson DJ. Development and feasibility of a scale to assess postoperative recovery: the post-operative quality recovery scale. 
Anesthesiology. 2010;113(4):892-905. Describes the Postoperative Quality of recovery scale.

35. Royse CF, Williams Z, Purser S, Newman S. Recovery after nasal surgery vs. tonsillectomy: discriminant validation of the Postoperative Quality of Recovery Scale. Acta Anaesthesiol Scand. 2014. doi:10.1111/aas.12264.

36. Johansen A, Romundstad L, Nielsen CS, Schirmer H, Stubhaug A. Persistent postsurgical pain in a general population: prevalence and predictors in the Troms $\varnothing$ study. Pain. 2012;153(7): 1390-6.

37. Werner MU, Bischoff JM. Persistent postsurgical pain: evidence from breast cancer surgery, groin hernia repair, and lung cancer surgery. Curr Top Behav Neurosci. 2014. doi:10.1007/7854_ 2014_285.

38. Aasvang EK, Gmaehle E, Hansen JB, Gmaehle B, Forman JL, Schwarz J, Bittner R, Kehlet H. Predictive risk factors for persistent postherniotomy pain. Anesthesiology. 2010;112(4): 957-69.

39. VanDenKerkhof EG, Peters ML, Bruce J. Chronic pain after surgery: time for standardization? A framework to establish core risk factor and outcome domains for epidemiological studies. Clin J Pain. 2013;29(1):2-8.

40. Chaparro LE, Smith SA, Moore RA, Wiffen PJ, Gilron I. Pharmacotherapy for the prevention of chronic pain after surgery in adults. Cochrane Database Syst Rev. 2013;7:CD008307. doi:10. 1002/14651858.CD008307.

41. Hovens IB, Schoemaker RG, van der Zee EA, Absalom AR, Heineman E, van Leeuwen BL. Postoperative cognitive dysfunction: involvement of neuroinflammation and neuronal functioning. Brain Behav Immun. 2014;38:202-10. doi:10.1016/j.bbi. 2014.02.002.

42. Jildenstål PK, Hallén JL, Rawal N, Berggren L, Jakobsson JG. AAI-guided anaesthesia is associated with lower incidence of 24-hour MMSE $<25$ and may impact the IL-6 response. Int J Surg. 2014;12(4):290-5. doi:10.1016/j.ijsu.2014.02.002.

43. - Chan MT, Cheng BC, Lee TM, Gin T; CODA Trial Group. BISguided anesthesia decreases postoperative delirium and cognitive decline. J Neurosurg Anesthesiol. 2013;25(1):33-42. Addressed the potential effects of reducing the occurrence of early postoperative cognitive side-effects from the use of BIS.
44. Brattwall M, Stomberg MW, Rawal N, Segerdahl M, Houltz E, Jakobsson J. Patient assessed health profile: a six-month quality of life questionnaire survey after day surgery. Scand J Public Health. 2010;38(6):574-9.

45. Velanovich V. Laparoscopic vs open surgery: a preliminary comparison of quality-of-life outcomes. Surg Endosc. 2000; 14(1):16-21.

46. Dhankhar DS, Sharma N, Mishra T, Kaur N, Singh S, Gupta S. Totally extraperitoneal repair under general anesthesia versus Lichtenstein repair under local anesthesia for unilateral inguinal hernia: a prospective randomized controlled trial. Surg Endosc. 2013;28(3):996-1002.

47. Errando CL, Aldecoa C. Awareness with explicit recall during general anaesthesia: current status and issues. $\mathrm{Br} \mathrm{J}$ Anaesth. 2014;112(1):1-4.

48. Strøm C, Rasmussen LS, Sieber FE. Should general anaesthesia be avoided in the elderly? Anaesthesia. 2014;69(Suppl 1):35-44.

49. Canet J, Raeder J, Rasmussen LS, Enlund M, Kuipers HM, Hanning CD, Jolles J, Korttila K, Siersma VD, Dodds C, Abildstrom H, Sneyd JR, Vila P, Johnson T, MuñozCorsini L, Silverstein JH, Nielsen IK, Moller JT, ISPOCD2 Investigators. Cognitive dysfunction after minor surgery in the elderly. Acta Anaesthesiol Scand. 2003;47(10):1204-10.

50. Krenk L, Rasmussen LS, Kehlet H. Delirium in the fast-track surgery setting. Best Pract Res Clin Anaesthesiol. 2012;26(3): 345-53.

51. Steinmetz J, Siersma V, Kessing LV, Rasmussen LS, ISPOCD Group. Is postoperative cognitive dysfunction a risk factor for dementia? A cohort follow-up study. Br J Anaesth. 2013;110(Suppl 1):92-7.

52. Green JS, Tsui BC. Impact of anesthesia for cancer surgery: continuing professional development. Can J Anaesth. 2013; 60(12):1248-69.

53. Cata JP, Hernandez M, Lewis VO, Kurz A. Can regional anesthesia and analgesia prolong cancer survival after orthopaedic oncologic surgery? Clin Orthop Relat Res. 2013;472(5):1434-41.

54. Swyers T, Redford D, Larson DF. Volatile anesthetic-induced preconditioning. Perfusion. 2014;29(1):10-5. 